\title{
Application of Nuclear Particle Tracks: A Scanning X-Ray Microscope
}

\author{
P. J. Ebert
}

This paper was prepared for submittal to the

7th National Conference on Synchrotron

Radiation Instrumentation

Baton Rouge, LA

October 28-31, 1991

September 30, 1991

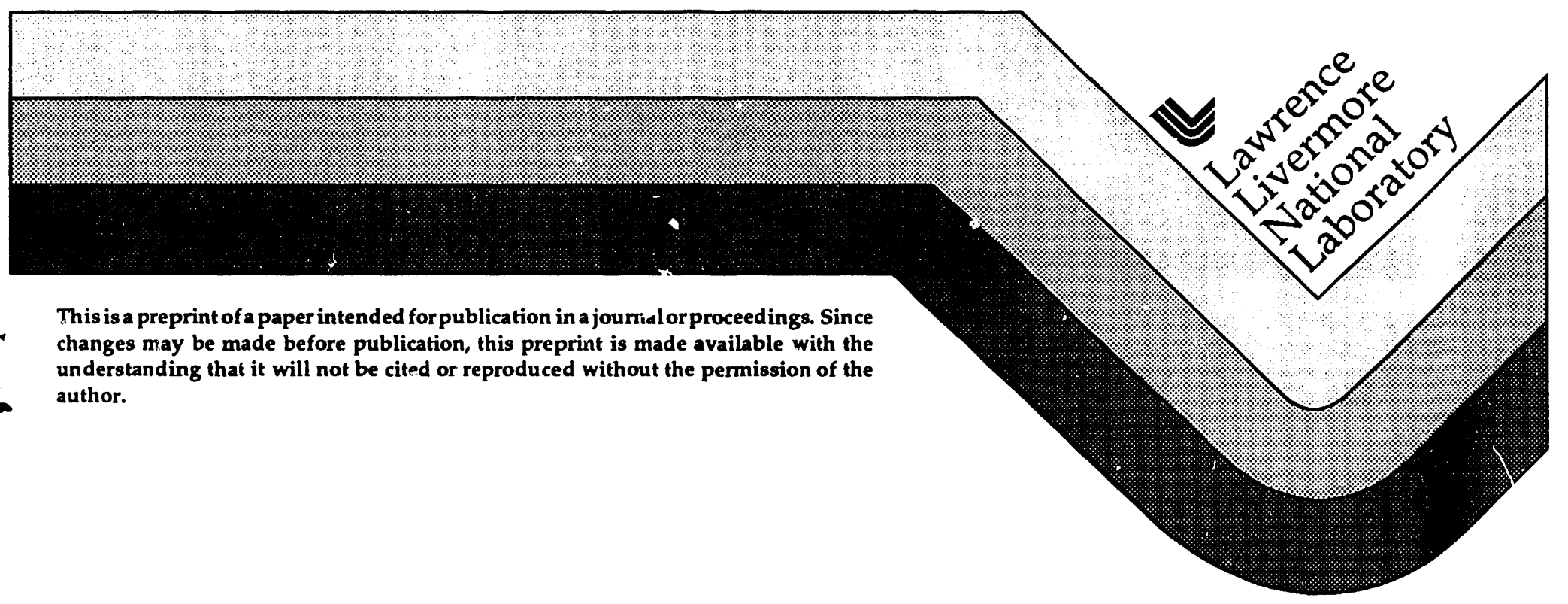

This is a preprint of a paper intended for publication in a jourrial or proceedings. Since changes may be made before publication, this preprint is made available with the understanding that it will not be cited or reproduced without the permission of the author. 


\section{DISCI.AIMER}

This document was prepared as an account of work sponsored by an agency of the United States Government. Neither the United States Government nor the University of Californiz nor any of their employees, makes any warranty, express or implied, or assumes any legai liability or responsibility for the accuracy, completeness, or usefulness of any information, apparatus, product. or process disclosed, or represents that its use would not infringe privately owned rights. Reference herein to any specific cummercial products. process, or service by trade name, trademark, manufacturer, or otherwise. does not necessarily constitute or imply its endorcement, recommendation. or favoring by the United States Government or the Liniversity of California. The views and opinions of authors expressed herein do not necessarily state or reflect those of the United States Government or the University of California, and shall not be used for advertising or product endorsement purposes. 


\title{
Application of nuclear particle tracks: a scanning $x$-ray microscope
}

\author{
Paul J. Ebert \\ University of California \\ Lawrence Livermore National Laboratory \\ Livermore, CA 94550
}

The scanning $x$-ray microscope (SXM) is a short-wavelength analog of a near-field optical-scanning microscope, promising spatial resolution of $\sim 100 \AA$ up to $\sim 5 \mathrm{keV} x$-ray energy. A portion of a synchrotron $\mathrm{x}$-ray beam streams through an etched nuclear particle track in an opaque membrane and impinges on an object within the narrow stream. Scattered or transmitted $x$-rays are detected with a photon counter. The SXM is feasible because a useful number of synchrotron $x$-rays, even from a bend magnet, will stream through a small diameter pore. The properties and limitations of the SXM are discussed together with other submicroscopic applications of nuclear particle tracks. 


\title{
Application of nuclear particle tracks: a scanning x-ray microscope
}

\author{
INTRODUCTION
}

The x-ray microscope is becoming an indispensable tool in many areas of scientific research, particularly in the life sciences. [1] Of greatest interest are $x$-rays that fall within the water window, the $265 \mathrm{eV}$ to $530 \mathrm{eV}$ energy (or $47 \AA$ to $23 \AA$ wavelength) range where water is transparent but other cell constituents are not. In a recent review four $x$-ray microscopy methods were described and the state-ofthe-art was assessed. [2] The scanning $x$-ray microscope (SXM) considered herein is a shori-wavelength analog of the near-field optical-scanning microscope. [3] It stems from the realization that a useful number of synchrotron $x$-rays, even from a bend magnet, will stream through a very small diameter hole.

The SXM promises spatial resolution of $\sim 100 \AA$ over a very broad energy range that extends to $\sim 5 \mathrm{keV}$, far outside the water window. This would be a considerable improvement over present-day imaging. As is schematically illustrated in Fig. 1, a portion of a synchrotron $x$-ray beam streams through a very small diameter pore (an etched nuclear particle track) in an opaque membrane and impinges on an object within the stream. The components of the object can be identified by either absorption or scattering. Fig. 1 shows resonantly scattered $x$-rays detected with a photon counter that subtends $2 \pi \mathrm{sr}$. With the shadow shield removed, transmitted $x$-rays would be detected. The SXM is ideal for computer data acquisition and image analysis even though scanning time may be long.

Very small diameter pores are produced by irradiating a dielectric with heavy ions and then dissolving the residual material along the ion's track. [4] Commercially available polycarbonate plastic films have various thicknesses and pore diameters, with the smallest pores having $\sim 100 \AA$ diameter. [5] Tracks of $6 \mu \mathrm{m}$ in length would be useful for forming images with $x$-rays within the water window to $\sim 400 \mathrm{eV}$. By monitoring and controlling heavy ion beam irradiation with a solid state detector, it should be possible to obtain single, isolated $100 \AA$ diameter pores in mica $100 \mu \mathrm{m}$ thick. [6] Tracks produced in this manner would be opaque to $\sim 3 \mathrm{keV} x$-rays. Very recently, Bursill and Braunshausen produced $140 \mu \mathrm{m}$ long latent or unetched tracks with $80 \AA$ diameters in zircon $\left(\mathrm{ZrSiO}_{4}\right)$. [7] It is not clear whether etched tracks would retain their diameters. However, a channel this long in zircon would collimate much higher energy ( $6 \mathrm{keV}) \mathrm{x}$-rays. See Fig. 2.

The SXM is conceptually very simple, and as shown below, it is feasible from a theoretical standpoint. It has several other closely related applications which derive largely from the inherent shielding of the opaque material. Limitations as well as straightforward $x$-ray optical measurements that would clearly confirm feasibility are also discussed. 
The SXM is theoretically feasible if the number of $x$-rays per second streaming through the hole is both countable and significantly greater than the number per second which pass through the foil material, that is

$$
R_{h}=\phi A_{h}>\phi A_{b} e^{-\mu x} .
$$

$\phi$ is the $x$-ray flux ( $x$-rays $/ \mathrm{cm}^{2} \mathrm{sec}$ ), assumed to be uniform; $A_{h}=\pi d_{h}{ }^{2} / 4$ is the area of the hole; $A_{b}$ is the area of the $x$-ray beam; $e^{-\mu x}$ is the fraction of beam $x$-rays transmitted through the foil material; $\mu$ is the linear absorption coefficient; $x$ is the length of the pore.

Values of $R_{h}$ from undulator, wiggler and bend magnet beam lines at several US synchrotrons are given in Table I. A useful rate is derived in every case. Note that for some applications, it would be better to use an $x$-ray beam that has wide inherent bandwidth, such as from a bend magnet or wiggler. Such beams would have higher $R_{h}$ values than are given in Table I. An optical component such as a toroidal mirror that collects and focuses $x$-rays can also be used to increase $R_{h}$ from a bend magnet or wiggler source. Thus, the SXM does not require a super-brilliant, quasi-coherent undulator source, and based on the considerations that have been presented so far, it is safe to conclude that the SXM consisting of a photon counter to detect $x$-rays that stream through a small diameter pore is feasible.

\section{FORMING AN IMAGE}

An object on a transparent mounting is rastered through the $x$-ray beam with piezoelectric transducers. For each dwell time, $x$-rays are counted with an efficient detector, typically a flow proportional counter, located behind the sample. An image is formed by counting a) $x$-rays transmitted through the object, b) fluorescence $x$-rays emitted by selected elements in the object, or c) $x$-1.ays that are resonantly scattered by specific elements in the object. To have comparable recording times, methods b) and $c)$ require considerably higher $R_{h}$ than method a). Method c) also requires much narrower line width than is given in Table $I$, and as a consequence, is more selective than methods $b$ ) and a).

The time required to record an image is governed by a number of factors: object size, desired resolution, and desired image contrast. As an example, consider the geometry that is schematically illustrated in Fig. 3. A $1 \mu \mathrm{m}$ diameter protein sphere is suspended in water and sandwiched between two $\mathrm{Si}_{3} \mathrm{~N}_{4}$ films $400 \AA$ thick. As is evident in Fig. 4, which shows transmittance as a function of radius, the sphere is significantly more opaque at $404 \mathrm{eV}$ (above the $\mathrm{K}$-edge of nitrogen) than at $400 \mathrm{eV}$ (below the $\mathrm{K}$ edge). The $\mathrm{Si}_{3} \mathrm{~N}_{4}$ films are also more opaque, but remain featureless because they have uniform thickness. With a $100 \AA$ diameter collimating pore that channels $2 \times 10^{4} \mathrm{x}$-rays/sec, it would take approximately 13 minutes to scan the object and obtain an image with 32 shades of gray (1000 counts). With a $200 \AA$ diameter channel, it would take approximately $50 \mathrm{sec}$ to obtain an image with 32 shades 
of gray. For a given object, the time to acquire an image is inversely proportional to the fourth power of channel diameter.

\section{FACTORS LIMITING PERFORMANCE}

Like other $x$-ray imagers, the SXM has limitations. Among the factors giving rise to limitations are locating and aligning the pore, $x$-ray diffraction, thermal loading and $x$-ray dose. They are discussed together with possible means of avoiding them in this section. Radiation dose is treated in some detail because this imaging approach differs from other approaches in this regard.

Locating and aligning the etched track

Owing to dielectric charging, a scanning electron microscope cannot be used to locate both entrance and exit apertures of an etched track. However, if the pore is normal to the surface of the foil and within the critical angle for $x$-ray specular reflection, it should be possible to align the pore using sensitive photon counting technique: the foil is mounted on a goniometer and the angle of incidence adjusted until the count rate is maximum. An alternative method would be to apply $x$-ray lithographic technique: the foil is backed with an etchable resist, scanned with $x$-rays and the resist overdeveloped to detect the hole location optically. The accuracy of this method would be limited by the optical resolution of the microscope.

\section{X-ray diffraction}

Resolution in scanning depends upon the location of the object with respect to the exit aperture of the pore. Assuming Fraunhofer diffraction, which is obtained at a distance $Z>d_{h}{ }^{2} / \lambda$, spatial resolution is given by $\Delta x \approx d_{h}+1.22 \lambda Z / d_{h}$, that is, the beam expands according to the Rayleigh diffraction criterion. The object should be located as close to the aperture as is possible, in the Fresnel diffraction region. Spatial resolution in scanning an object located in front of the pore would not be subject to this limitation. In this configuration, all of the object would be exposed to $x$-rays. See Fig. 5 . It should be noted that the depth of focus of a very thin Fresnel lens would be comparable to $d_{h}{ }^{2} / \lambda$.

\section{Thermal loading}

All $x$-ray imagers that use synchrotron beams are subject to thermal loading. The $x$-ray heat load in watts/gm is given by $\phi \mathrm{h} v(\mu / \rho)$, where $\phi \mathrm{h} v$ is the $\mathrm{x}$-ray energy flux (watts/ $\mathrm{cm}^{2}$ ) and $(\mu \rho)$ is the mass absorption coefficient $\left(\mathrm{cm}^{2} / \mathrm{g}\right)$. It can be reduced considerably for the SXM with a precollimator and, for the case of high brilliance undulator sources which result in beams that are too intense to count, with spaced attenuator foils and a precollimator. A precollimator also reduces $A_{b}$ considerably. See equation (1). 
X-ray dose

In microscopy with ionizing radiation, the dose delivered to an object increases with higher resolution and contrast because more photons per unit area are necessary to improve either spatial resolution or image contrast. Of particular concern is killing a cell or destroying a biological function while an image is being made. [8] Indeed, the radiation dose delivered by an $x$-ray laser to obtain a Gabortype hologram will be so large $\left(\sim 10^{10} \mathrm{Rad}\right)$ that there is no hope of cell survival. $[8,9]$

The SXM dose is smaller for two reasons: first, unlike holography which requires detection of scattered $x$-rays, the scanned image records transmitted $x$-rays, and requires far fewer photons; second, only a very small part of the cell is exposed at any time. Even so, a scanned cell would receive a lethal radiation dose, as shown below.

To appreciate this consequence of submicroscopic imaging with $x$-rays, we estimate of the dose delivered to a small part of a cell by $x$-rays streaming through a $100 \AA$ diameter hole. In the usual dose calculation, dose in Rad is given by the expression

$$
D=\phi t h v(\mu / \rho) \times 1.6 \times 10^{-11}
$$

where $t$ is exposure time; $h v$ is $x$-ray energy (keV). In (2) it is implicitly stated that photon energy is deposited locally, at the point of interaction; equivalently, in every volume element of the exposed object, "in" scattering of electrons and photons are equal to "out" scattering. Thus, equation (2) is valid when both photon mean free path and beam radius are very large compared with the ranges of secondary electrons and the dimensions of the exposed object.

For the SXM, exposure conditions are different, and electron ranges should be taken into account because they are not negligible compared to the beam radius. Dose is the secondary electron energy divided by the excited mass, which is contained in a cylinder with height equal to the photon mean-freepath and radius equal to the electron range plus the radius of the beam. Although photoelectrons are ejected mainly in the plane of polarization of the incident x-rays, they do not travel in straight paths, but are scattered and follow random paths. Strictly speaking, their range is the path length traveled in slowing down, but for dose calculation, electron range is the thickness of a flat slab which absorbs nearly all of the electron energy. This is much less than the slowing-down range. The kinetic energy of photoelectrons is given by $T_{P E}=h v-B_{K}$, where $B_{K}$ is the K-electron binding energy. In $C, N$ and $O$, we ignore $B_{L}$, the $L$-shell binding energy. Thus, Auger electron energy is given by $T_{A E} \approx B_{K}$. Electron range in $\AA$ is assumed to increase linearly with energy according to the expression

$$
R_{A E, P E}=0.4 C T_{A E, E}+20,
$$

where $\mathrm{T}$ is in $\mathrm{eV}$. Equation (3) was obtained from straight line fits to ranges of 50 to $600 \mathrm{eV}$ electrons in organic materials with unit density. [10,11] Densities and atomic fractions of cell materials were taken from London et al. [9] With these assumptions, the dose in Rad can then be written as 
A Scanning $X$ ray Microscope

$$
D=N \boldsymbol{\Sigma}_{i j}\left[f_{i}(\mu / \rho)_{i}\left(A_{i} / \underline{\underline{A}}\right)\left(\boldsymbol{\Sigma}_{j} F_{i j}\left(T_{e}\right)\right]\right] \times 1.6 \times 10^{-11}
$$

In (4), $N$ is the number of $x$-rays streaming through the hole, $f_{i}$ is the atomic fraction and $(\mu / \rho)_{i}$ is its corresponding mass absorption coefficient, $A_{i}$ is the corresponding atomic weight and $\underline{\underline{A}}$ is the average atomic weight of the cell constituent material (eg protein, DNA, lipid, nucleosome). The sum $\boldsymbol{\Sigma}_{\mathrm{j}} \mathrm{F}_{\mathrm{ij}}$ accounts for non-local electron energy deposition, and is given by

$$
F_{i j}\left(T_{e}\right)=\rho^{2}\left[T_{i P E} / \pi\left(R_{P E}+R_{C}\right)^{2}+T_{i A E} / \pi\left(R_{A E}+R_{C}\right)^{2}\right]
$$

$R_{C}$ is the radius of the pore and $\rho$ is density of the material in the cell. In this approximation, it is assumed that photoelectric absorption is with K-shell electrons, and that the vacancy is filled by Auger electron emission. This is not unreasonable for low $Z$ elements. The dose to protein from $1000 x$-rays streaming through a $100 \AA$ diameter hole is plotted as a function of $x$-ray energy in Fig. 6 . The solid squares were obtained with equation (2) and the open squares using equations (3), (4) and (5). In Fig. 7 doses to other cellular constituents obtained with equations (3), (4) and (5) are compared with that delivered to protein. There is little doubt that a living cell would not survive scanning with 1000 photons per $100 \AA$ diameter pixel. Mortality is a consequence of obtaining a high resolution image; a large radiation dose is delivered because a fairly high energy flux is absorbed by a very smali mass. However, with the SXM, it would also be possible to scan a very small part of a cell without exposing the entire cell to a lethal radiation dose; under such circumstances, the cell rught survive even though the irradiated component might be damaged.

In this connection, it is both interesting and instructive to consider the $\mathrm{x}$-ray dose delivered by a single photon. For this estimate, divide equation (4) by $N$ and let $R_{C}=0$ in equation (5). The excited volume is now a cylinder whose radius is approximately equal tc the electron range and whose height is equal to the $x$-ray mean-free-path. While this is the correct height for a large number of photon interactions, it is much too large for interaction of a single $x$-ray, which is probably absorbed within the first mean-free-path in a smaller volume. Although the values obtained are too low, it is clear, from Fig. 8 that even a single soft $x$-ray packs quite a radiation dose.

\section{OTHER CLOSELY RELATED APPLICATIONS}

In addition to the SXM, there are a number of closely related applications of etched nuclear particle tracks. Based on the dose considerations given above, new, submicroscopic radiobiology experiments with live cells and ultrasoft $x$-rays are possible. Unique in this respect is the prospect of controlling exposure location (e.g. selected irradiation of mitocondria) together with photon energy, atomic species, number of $x$-rays, time of radiation (in the cell cycle), and $x$-ray polarization. The very small diameter $x$-ray beams could also be useful in submicroscopic dosimetry research. 
Of course, because photon counting is a very sensitive measurement technique, study of material composition at high spatial resolution is possible. Here, etched tracks in zircon or perhaps a dielsctric with higher atomic number and density might enable scanning with $\sim 10 \mathrm{keV} x$-rays. Highly localized excitation and charge generation are also possible.

Etched particle tracks should have bores which are very smooth compared to many surfaces. Thus, cylindrical and conical tracks should be useful $x$-ray light pipes or focusing devices for submicroscopic x-ray tomography. $[12,13]$

Another interesting application would be production of submicroscopic sources of visible light. Here, organic or inorganic scintillation material would be injected into the pores and excited by a shorter wavelength UV or $x$-ray source. [14]

\section{X-RAY OPTICAL EXPERIMENTS}

Feasibility of the SXM would be confirmed with results of straightforward $x$-ray diffraction experiments. These could be accomplished by measuring the angular distribution of the channeled $x$ ray beam with a photon counter. The counter would be located so that a Fraunhofer diffraction pattern and not an image of the $x$-ray source is obtained. The angular width of the diffraction, $\Delta \theta$, should be given by the Rayleigh diffraction criterion, $\Delta \Theta=1.22 \lambda / \mathrm{d}_{h}$. Diffraction measurements would also give insight into the possibility of obtaining depth information in experiments which apply resonant scattering or fluorescence to scan very small objects (See Fig. 1). It would be both interesting and important to experiment with tracks of different diameter and length in different materials and with different $x-$ ray energies.

This work was performed under the auspices of the U.S. Department of Energy by the Lawrence Livermore National Laboratory under Contract No. W-7405-ENG-48. 


\section{REFERENCES}

1. D. Attwood and B. Barton, Eds. "X-ray imaging for the life sciences-Proceedings of the Workshop," LBL 27660 (1989). CONF-8905192.

2 Malcolm R. Howells, Janos Kirz and David Sayre, "X-ray Microscopes," Scientific American, February 1991, p. 88. Also see references cited therein.

3. U. Durig, D. W. Pohl and F. Rohner, "Near-field optical-scanning microscopy," J. Appl. Phys. $\underline{59}$ 3318 (1986).

4. Robert L. Fleischer, P. Buford Price and Robert M. Walker, Nuclear Tracks in Solids, (University of California Press, Berkeley, 1975).

5. Poretics Corp., Livermore, CA and Nucleopore Corp., Pleasanton, CA

6. P. Buford Price, University of California. Private communication. 1991.

7. L. A. Bursill and Gerhard Braunshausen, "Heavy-ion irradiation tracks in zircon," Philosophical Magazine A 62,295 (1990).

8. See, for example R. M. Glaeser, "Limitations to significant information i . microscopy of biological macromolecules as a consequence of radiation damage," in "X-ray Microimaging for the Life SciencesProceedings of the Workshop," D. Attwood and B. Barton, Eds. LBL 27660 (1989). CONF-8905192.

9. R. A. London, M. D. Rosen and J. E. Trebes, "Wavelength choice for soft x-ray laser holography of biological samples," Applied Optics 28,3397 (1989). Also see p. 51 of Ref. 1.

10. A. Cole, "Absorption of $20 \mathrm{-eV}$ to $50,000-\mathrm{eV}$ electron beams in air and plastic," Radiation Research 38, 7 (1969).

11. J. C. Ashley, C. J. Tung and R. H. Ritchie, "Inelastic interactions of electrons with polystyrene: calculations of mean free paths, stopping powers, and csda ranges," IEEE Trans. Nucl. Sci. NS-25 1566 (1978).

12. E. A. Stern, Z. Kalman, A. Lewis and K. Lieberman, "Simple method for focusing $x$ rays using tapered capillaries," Applied Optics 2Z 5135 (1988).

13. K. Furuta, Y. Nakayama, M. Shoje, H. Nakano and Y. Hosokawa, "Intensity of $x$-ray microbeam formed by a hollow glass pipe," Rev. Sci. Instrum. $\underline{62} 828$ (1991).

14. K. Lieberman, S. Harush, A. Lewis and R. Kopelman, "A light source smaller than the optical wavelength," Science 247 59 (1990). Also see p. 166 of Ref. 1. 
A Scanning X-ray Microscope

TABLE I. Synchrotron radiation outputs: X-rays per sec through a $100 \AA$ diameter hole

\begin{tabular}{|c|c|c|c|c|c|c|}
\hline Ring & $\mathrm{E}-\mathrm{GeV}$ & $1-m A$ & Beam line & $h v \pm \Delta h v-e V$ & $\mathrm{Z}$-meters & $R_{h}-x$-rays $/ \mathrm{sec}$ \\
\hline$\overline{S S R L^{a}}$ & 3.0 & 100 & $\begin{array}{l}\text { V; } 30 \text { pole Und. } \\
\text { Bend }{ }^{\mathrm{b}}\end{array}$ & $\begin{array}{c}400 \pm 2 \\
4000 \pm 40\end{array}$ & - & $\begin{array}{l}\sim 10^{4} \\
\sim 10^{3}\end{array}$ \\
\hline $\mathrm{ALS}^{\mathrm{c}}$ & 1.5 & 400 & $\begin{array}{l}\text { U5; U3.9 } \\
\text { Bend }\end{array}$ & $\begin{array}{l}400 \pm 4 \\
400 \pm 4\end{array}$ & $\begin{array}{l}15 \\
10\end{array}$ & $\begin{array}{l}\sim 10^{7} \\
\sim 10^{3}\end{array}$ \\
\hline CAMDd & 1.2 & 400 & $\begin{array}{l}2 \mathrm{~m}, \mathrm{~K}=1 \text { Und. } \\
\text { Bend }\end{array}$ & $\begin{array}{l}400 \pm 4 \\
400 \pm 4\end{array}$ & $\begin{array}{l}15 \\
10\end{array}$ & $\begin{array}{l}\sim 10^{6} \\
\sim 10^{2}\end{array}$ \\
\hline APSe & 7 & 100 & $\begin{array}{l}\text { Und. A } \\
\text { Wig. A } \\
\text { Bend }\end{array}$ & $\begin{array}{l}4000 \pm 40 \\
4000 \pm 40 \\
4000 \pm 40\end{array}$ & $\begin{array}{l}25 \\
25 \\
25\end{array}$ & $\begin{array}{l}\sim 10^{8} \\
\sim 10^{4} \\
\sim 10^{2}\end{array}$ \\
\hline
\end{tabular}

a. R. Z. Bachrach. Private communication. 1991.

b. P. J. Ebert and C. J. Anderson, UCID 20458. Unpublished LLVL report.

c. A. Jackson, Nucl. Instrum. Meth. B 56/57 375 (1991).

d. R. L. Stockbauer, et al, Physica Scripta 41,788 (1990).

e. P. J. Viccaro, SPIE 1345, 28 (1990). 


\section{FIGURE CAPTIONS}

Figure 1. Schematic diagram of a scanning $x$-ray microscope. Synchrotron $x$-rays stream through a small diameter pore and impinge on a small object in the stream. Resonantly scattered or fluorescence $x-$ rays are detected with a photon counter that subtends $\sim 2 \pi$ sr. The shadow shield absorbs the narrow beam.

Figure 2. Transmittance of mica $\left(\mathrm{KAl}_{3} \mathrm{Si}_{3} \mathrm{O}_{12} \mathrm{H}_{2}\right)$, synthetic mica $\left(\mathrm{KMg}_{3} \mathrm{AlSi}_{3} \mathrm{O}_{10} \mathrm{~F}_{2}\right)$ and zircon $\left(\mathrm{ZrSiO}_{4}\right)$ foils $100 \mu \mathrm{m}$ thick.

Figure 3. Schematic diagram of scanning using $x$-ray transmission. A cell constituent (protein sphere) is suspended in water.

Figure 4. Transmittance of a $1 \mu \mathrm{m}$ diameter protein sphere suspended in water as a function of sphere radius. The $\mathrm{x}$-ray energies are just below $(400 \mathrm{eV})$ and just above $(404 \mathrm{eV})$ the $\mathrm{K}$-absorption edge of nitrogen. Transmittance at the center of the sphere decreases by a factor of $\sim 3$ with this small change in energy. Nitrogen is much more "visible."

Figure 5. Scanning with a narrow channel can result in diffraction and loss of spatial resolution. This effect can be reduced by locating the object in front of the foil (a.) rather than behind (b.) the foil. However, the dose will be much greater with the sample in front of the foil.

Figure 6. Comparison of estimated doses delivered to cellular protein by $1000 \mathrm{x}$-rays streaming through a $100 \AA$ diameter pore according to equation (2), assuming local energy deposition (solid squares) and according to equations (3), (4) and (5) assuming non-local energy deposition (open squares).

Figure 7. Estimated doses delivered by $1000 \mathrm{x}$-rays streaming through a $100 \AA$ diameter pore to different cellular materials. Equations (3), (4) and (5) were used to obtain these estimates.

Figure 8. Estimated dose delivered by a single $x$-ray to different cellular materials. The calculation assumes non-local deposition of energy. 


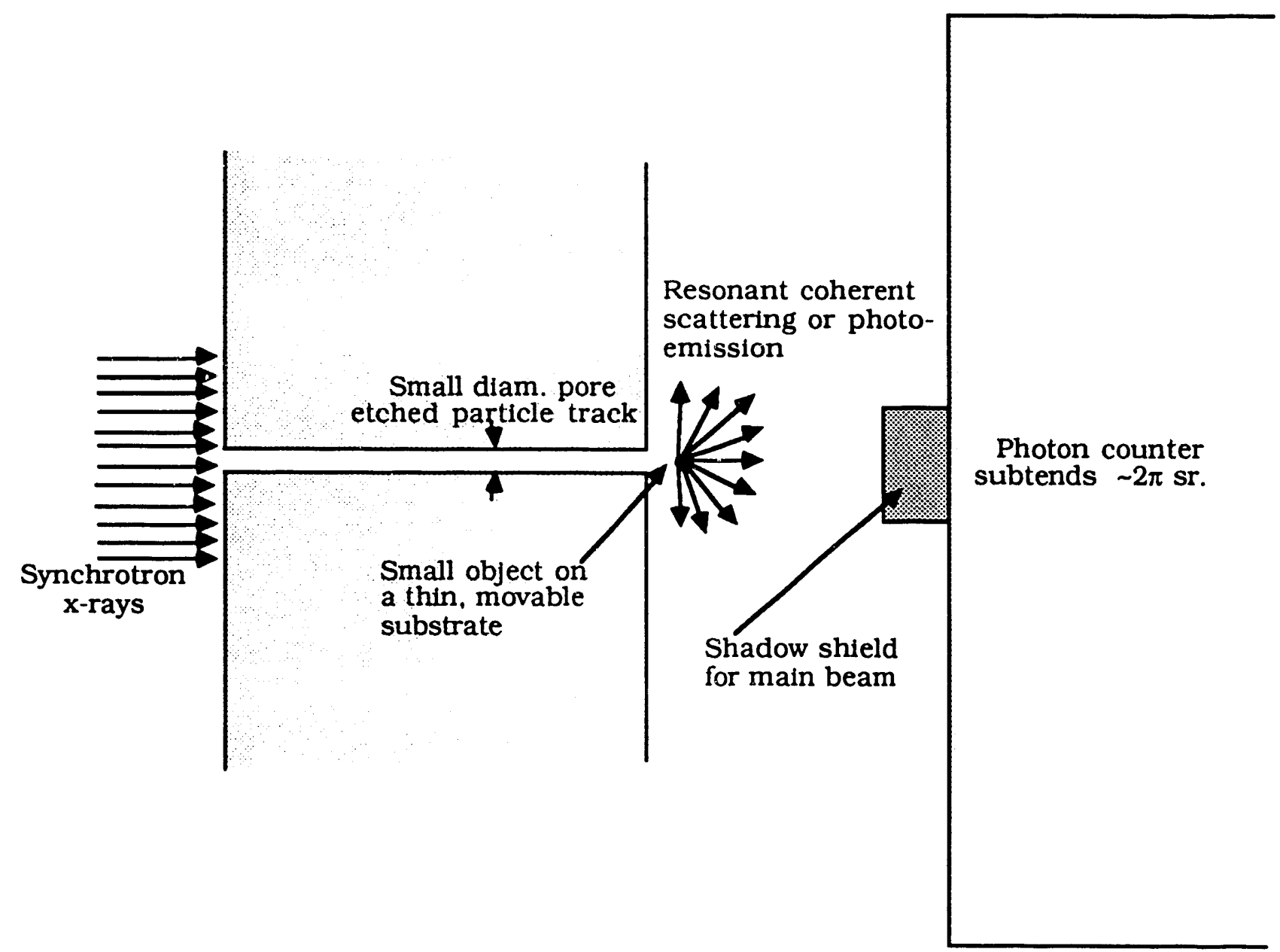

Figure 1. 
A Scanning X-ray Microscope

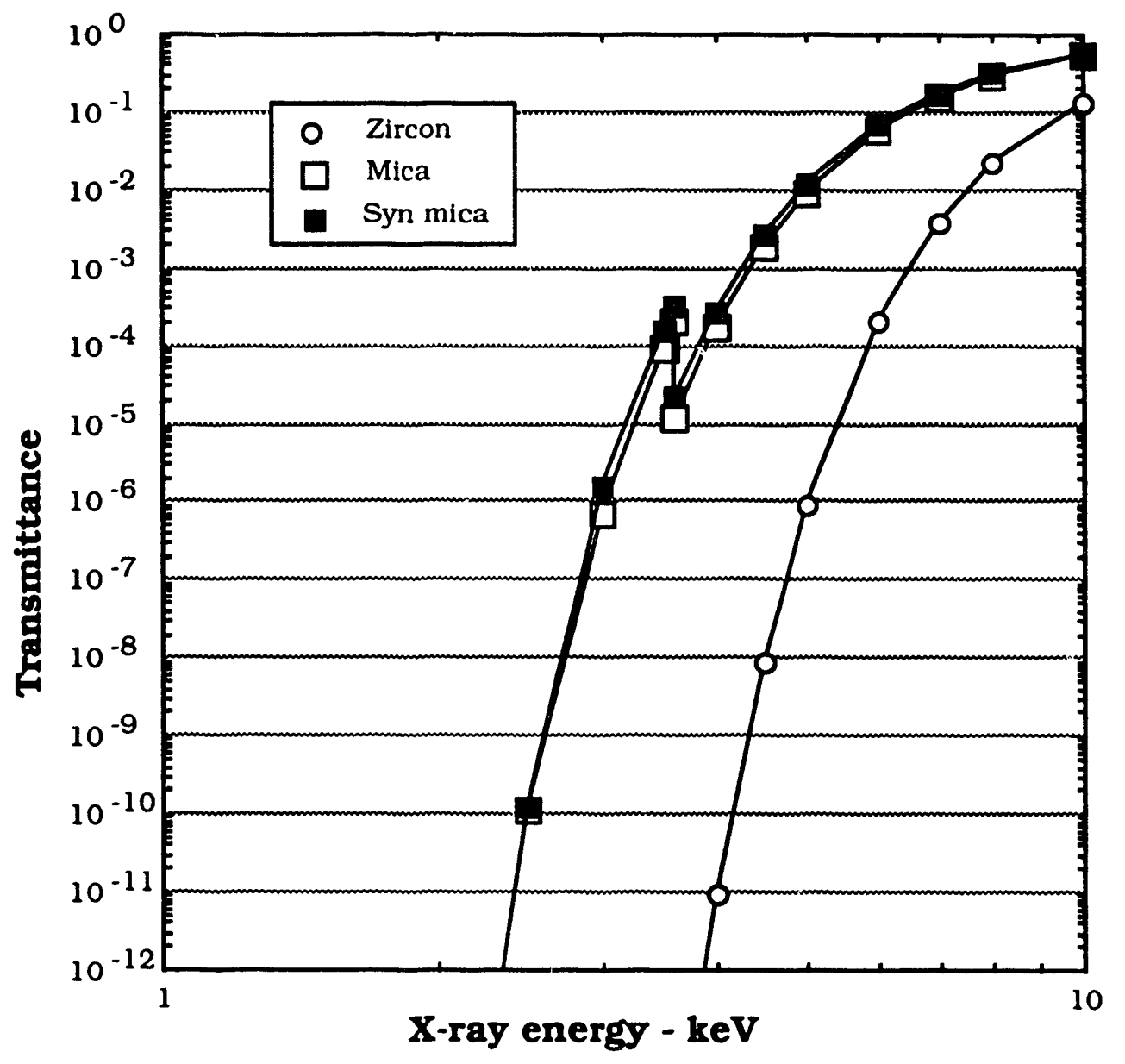

Figure 2. 


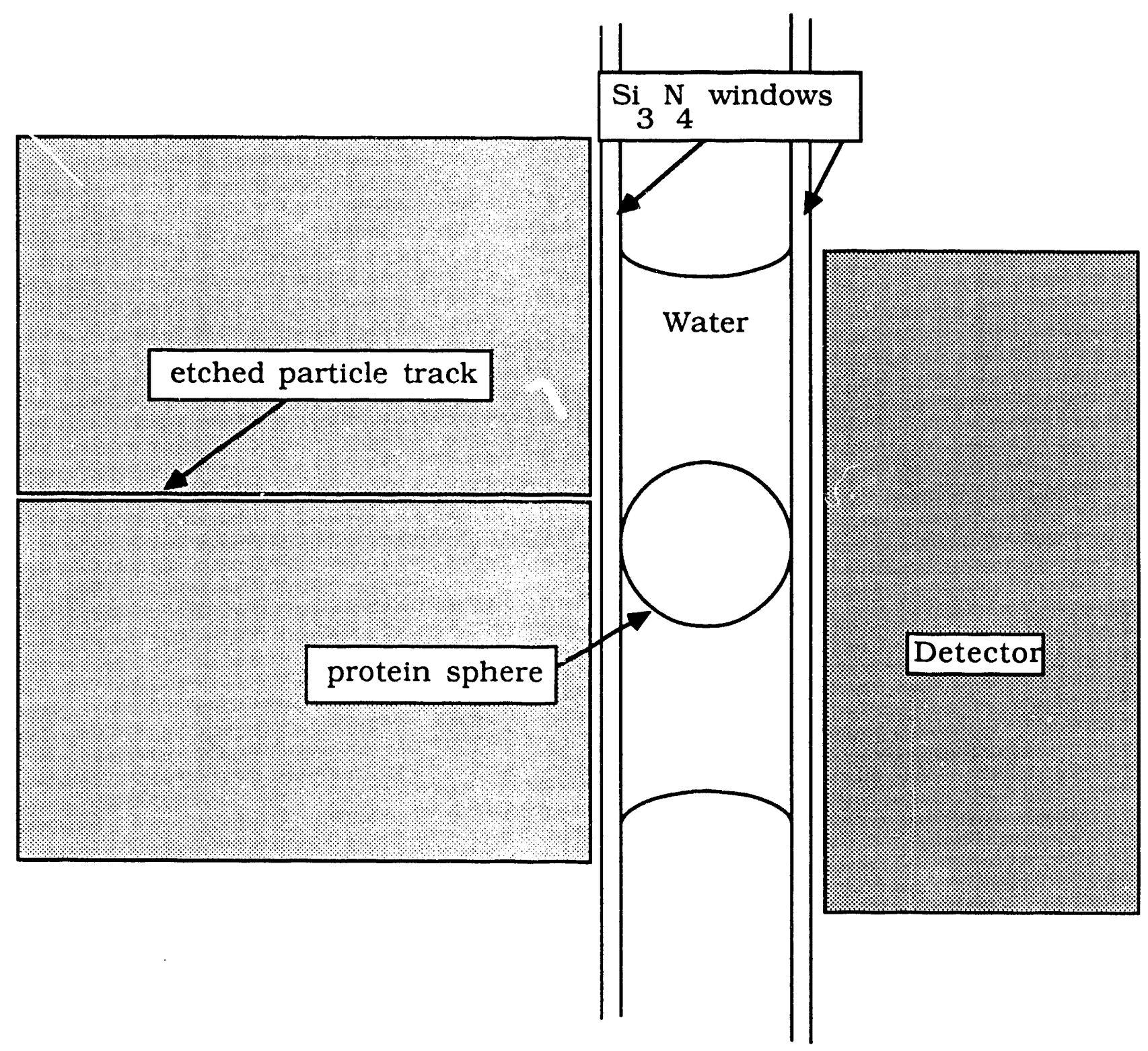

Figure 3. 
A Scanning X-ray Microscope

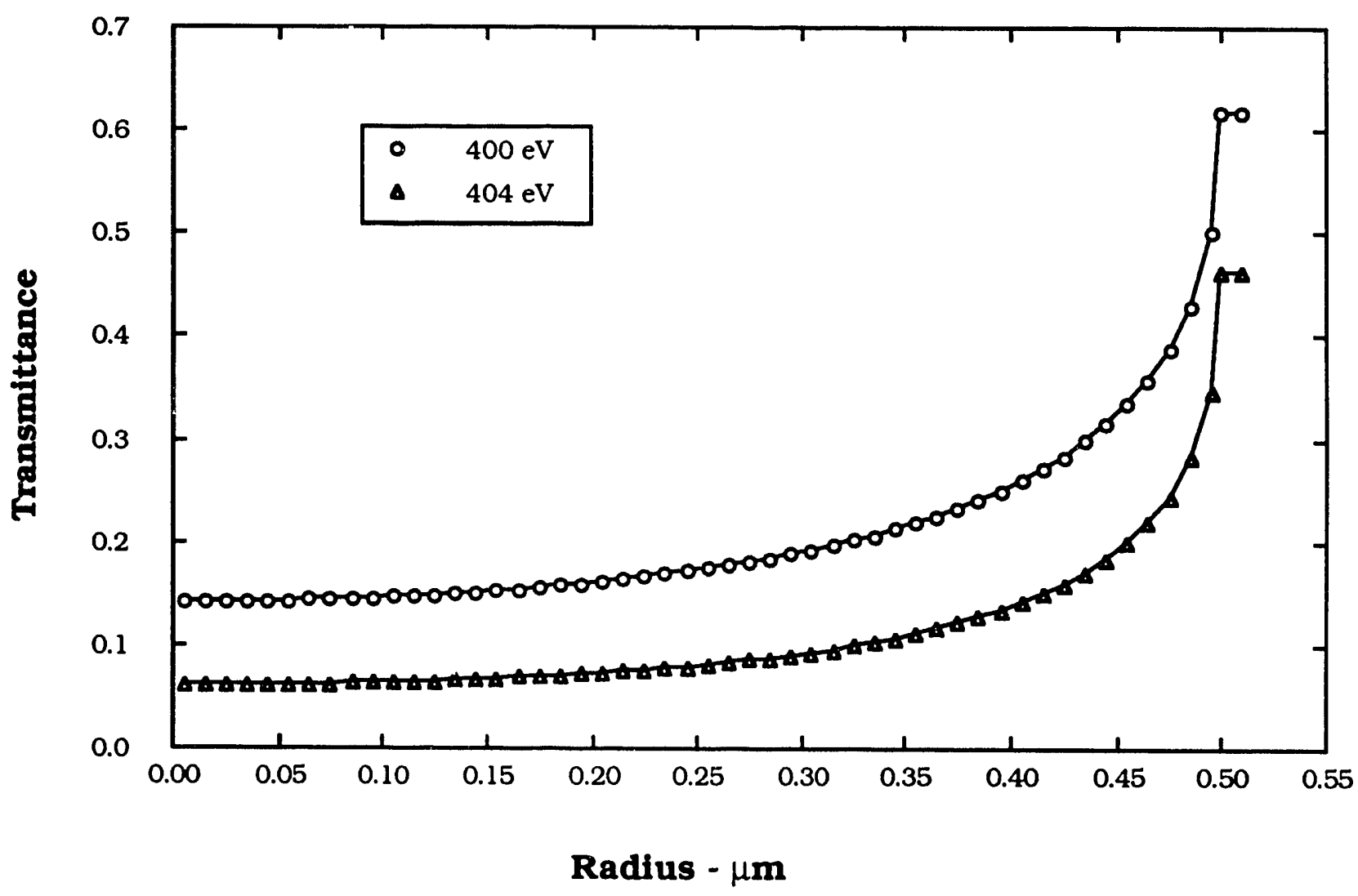

Figure 4 . 
A Scanning X-ray Microscope

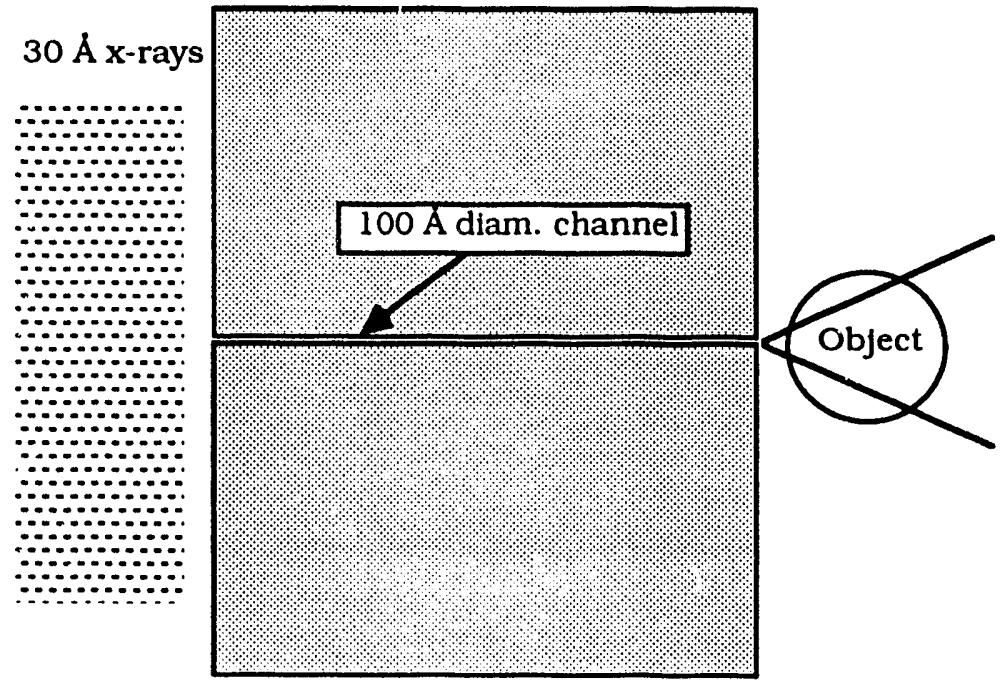

a

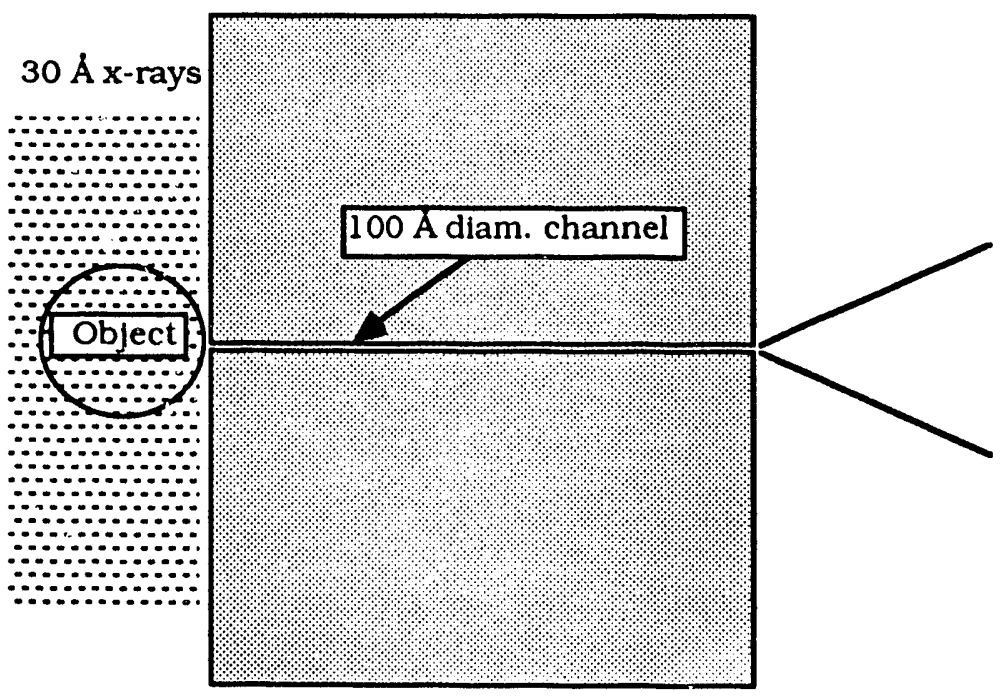

b.

Figure 5. 


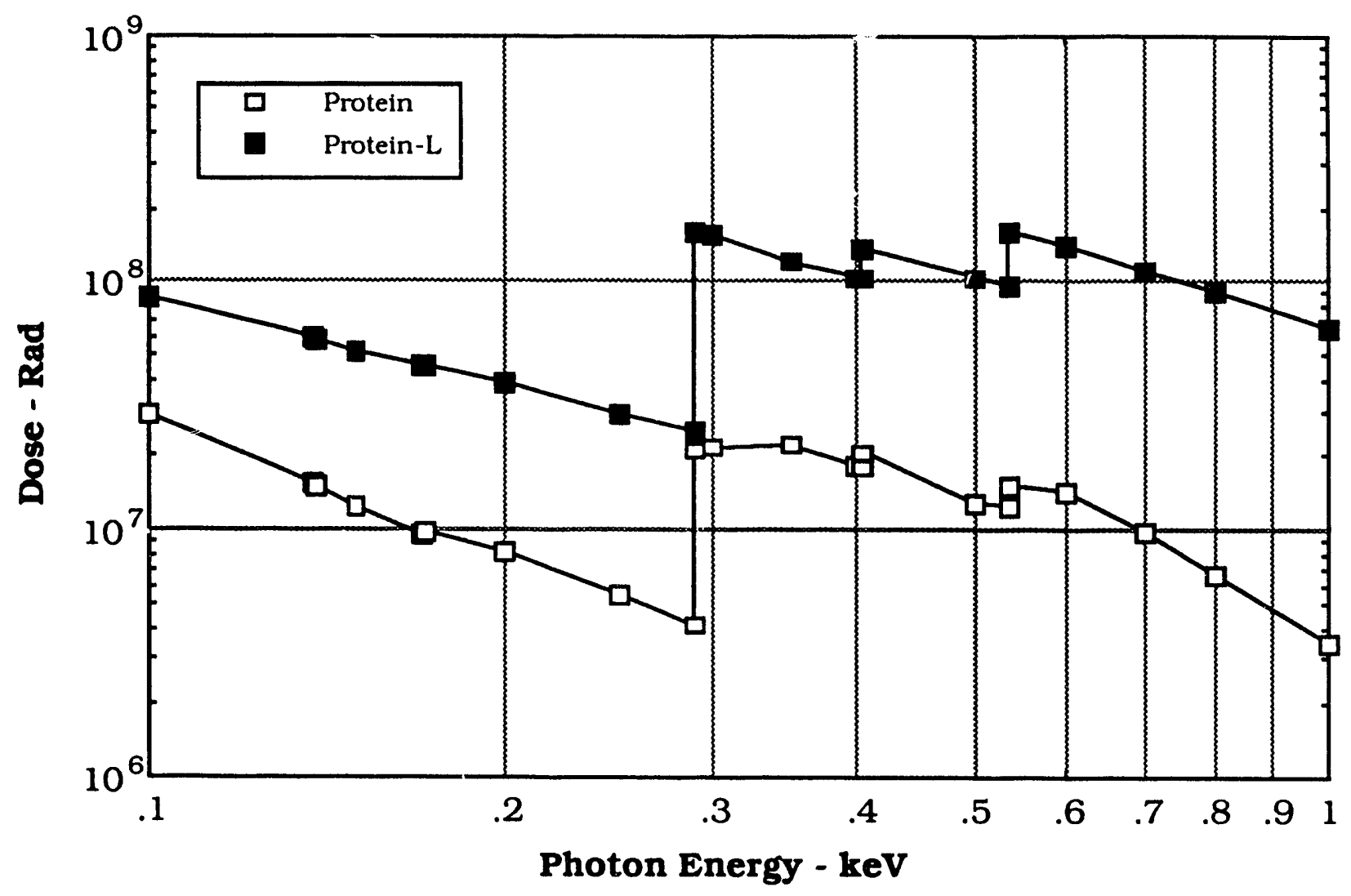

Figure 6. 


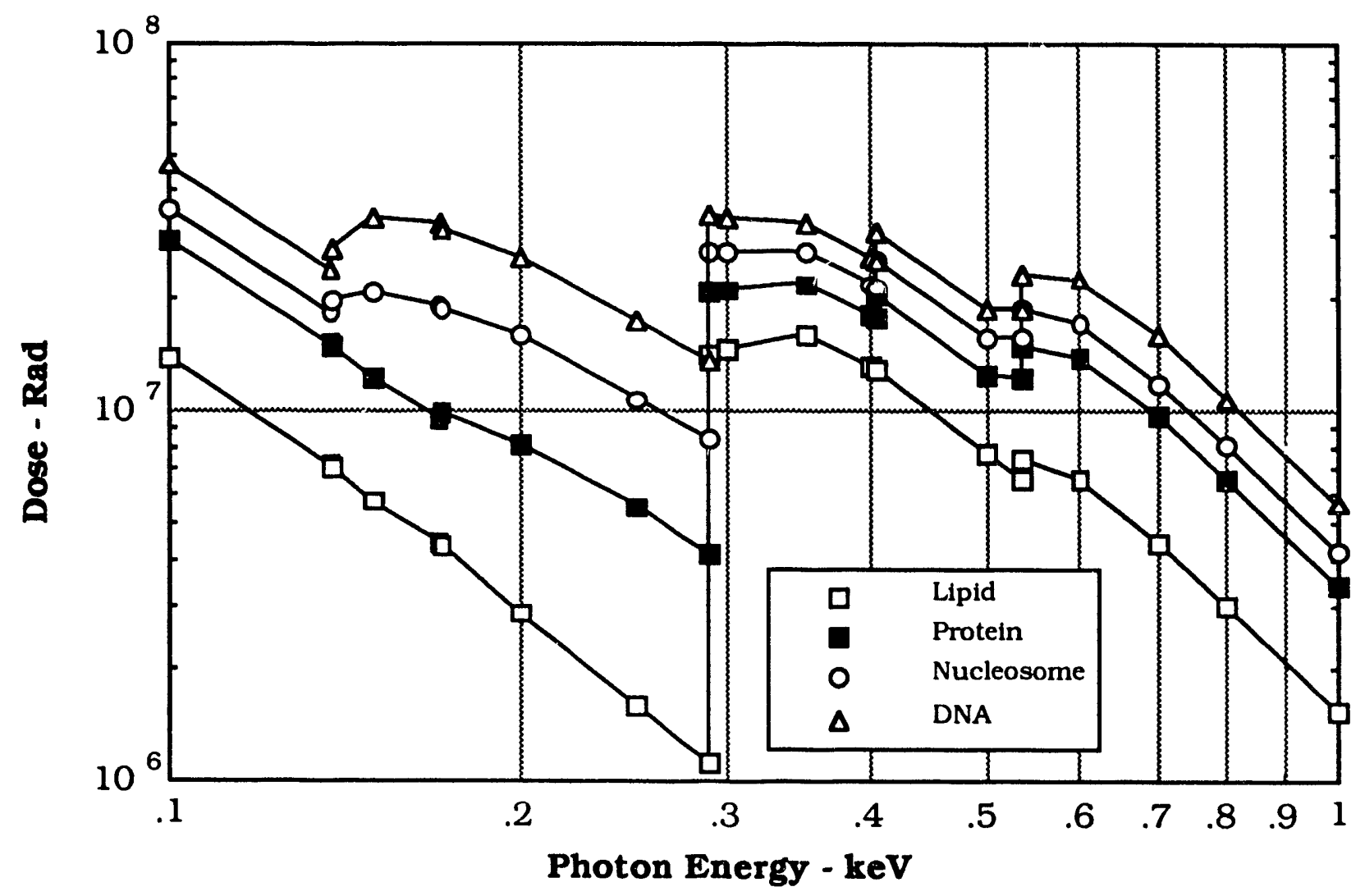

Figure 7. 
A Scanning X-ray Microscope

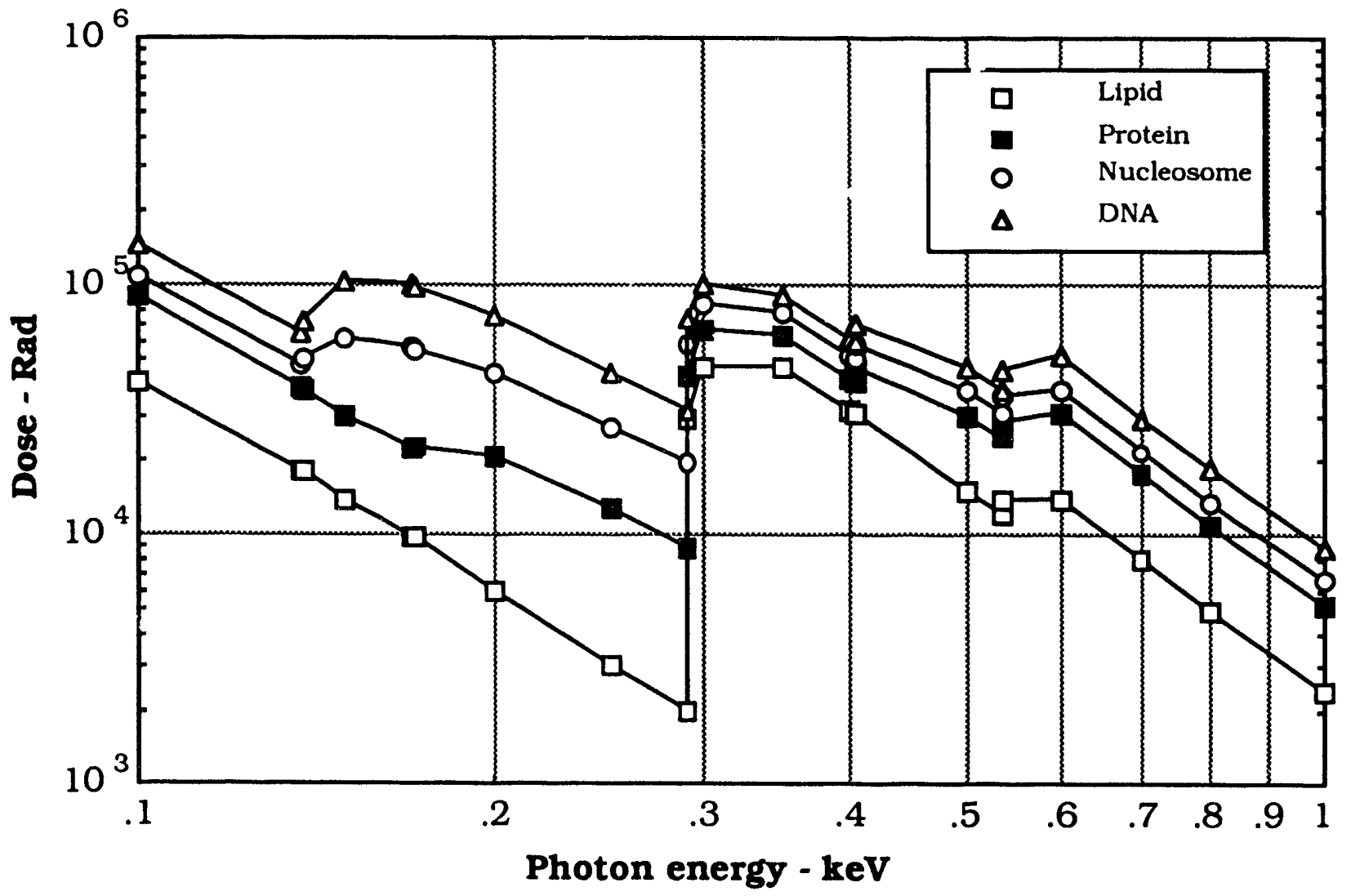

Figure 8. 

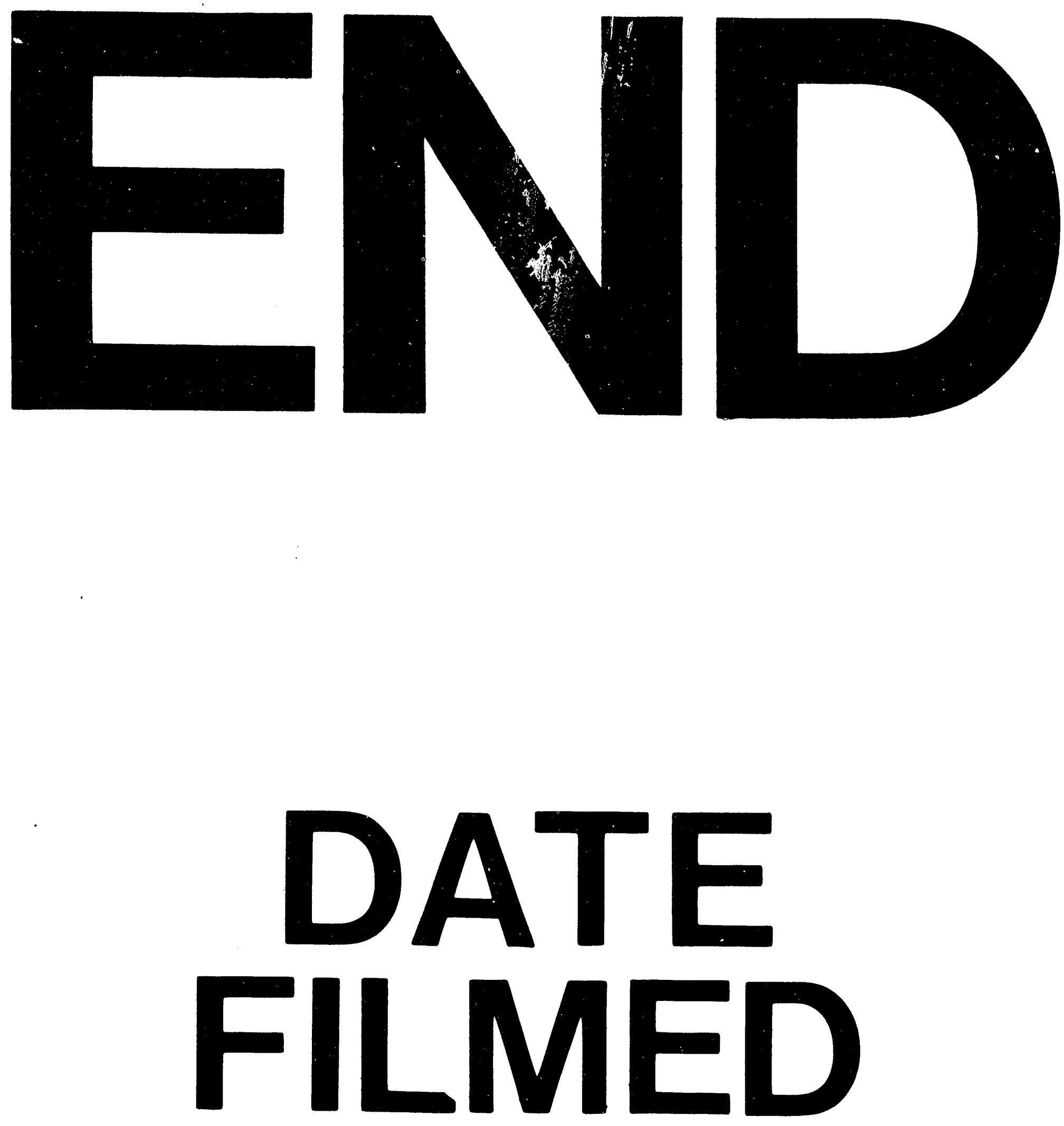

1

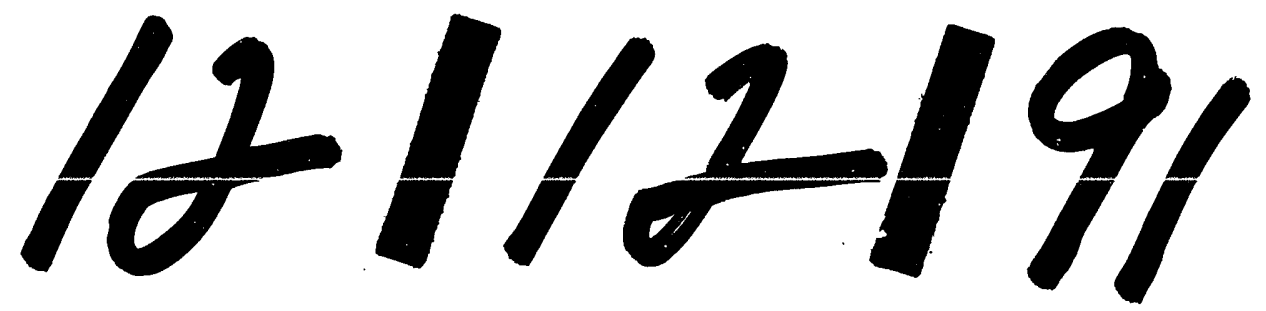

$\bar{I} i$ 
